\title{
Characterization of foreign materials in paraffin-embedded pathological specimens using in situ multi-elemental imaging with laser spectroscopy
}

Benoit Busser $^{1,2,3}$, Samuel Moncayo ${ }^{3}$, Florian Trichard ${ }^{3}$, Vincent Bonneterre ${ }^{2}$, Nicole Pinel ${ }^{2}$, Frédéric Pelascini ${ }^{4}$, Philippe Dugourd ${ }^{3}$, Jean-Luc Coll ${ }^{1}$, Michel D’Incan ${ }^{5}$, Julie Charles ${ }^{1,2}$, Vincent Motto-Ros ${ }^{3,6}$ and Lucie Sancey ${ }^{1,6}$

${ }^{1}$ Institute for Advanced Biosciences, UGA/Inserm U 1209/CNRS UMR 5309 joint research center, Grenoble, France; ${ }^{2}$ Grenoble Alpes University Hospital, Grenoble, France; ${ }^{3}$ Institut Lumière Matière, UMR 5306, Université Claude Bernard Lyon 1, CNRS, Villeurbanne, France; ${ }^{4}$ CRITT Matériaux Alsace, Schiltigheim, France and ${ }^{5}$ University Hospital of Clermont-Ferrand, Clermont-Ferrand, France

\begin{abstract}
Pathologists typically encounter many disparate exogenous materials in clinical specimens during their routine histopathological examinations, especially within the skin, lymph nodes, and lungs. These foreign substances may be free extracellular deposits or induce several clinical abnormalities or histopathological patterns. However, pathologists almost never investigate or report the chemical nature of exogenous metals in clinical specimens due to a lack of convenient and available technologies. In this paper, a novel strategy based on laserinduced breakdown spectroscopy (LIBS) technology is evaluated for in situ multi-elemental tissue imaging. The improved procedures allow visualization of the presence of chemical elements contained within paraffinembedded specimens of medical interest with elemental images that are stackable with conventional histology images. We selected relevant medical situations for which the associated pathology reports were limited to the presence of lymphohistiocytic and inflammatory cells containing granules (a granuloma and a pseudolymphoma) or to lymph nodes or skin tissues containing pigments or foreign substances. Exogenous elements such as aluminum, titanium, copper, and tungsten were identified and localized within the tissues. The all-optical LIBS elemental imaging instrument that we developed is fully compatible with conventional optical microscopy used for pathology analysis. When combined with routine histopathological analysis, LIBS is a versatile technology that might help pathologists establish or confirm diagnoses for a wide range of medical applications, particularly when the nature of external agents present in tissues needs to be investigated.
\end{abstract}

Modern Pathology (2018) 31, 378-384; doi:10.1038/modpathol.2017.152; published online 17 November 2017

Pathologists typically encounter many disparate exogenous materials in clinical specimens during their routine histopathological examinations. These various foreign substances may originate from voluntary causes (eg, tattoos or cosmetic fillers) or from involuntary causes (eg, accidental inclusion of external substances after trauma, surgery, and

Correspondence: Dr B Busser, PharmD, PhD, Institute for Advanced Biosciences, UGA/Inserm U 1209/CNRS UMR 5309 joint research center, Site Santé-Allée des Alpes, 38700 Grenoble, France.

E-mail: bbusser@chu-grenoble.fr

${ }^{6}$ These authors contributed equally to this work.

Received 1 August 2017; revised 14 September 2017; accepted 14 September 2017; published online 17 November 2017 ingestion or inhalation of particulate materials). ${ }^{1}$ Histopathologically, most of these exogenous agents may generate immune, inflammatory, or granulomatous reactions. Exogenous agents might also be simply encountered as free extracellular agents or particles in the tissues. Microscopic morphological examination of exogenous materials is helpful, but not always sufficient, to identify the nature of these foreign substances on the basis of a characteristic appearance and birefringence patterns, usually described as being particles, pigments, granules, vacuoles, fibers, or fragments of various forms, sizes, and colors. ${ }^{2}$ In some cases, pathologists may need to investigate the composition of the suspect foreign material to obtain or confirm a diagnosis or simply to complete their pathology report. 
A number of strategies might be used by pathologists to characterize the elemental composition of such exogenous substances, including histochemical staining for metals, microprobe X-ray imaging, or mass spectrometry imaging techniques (for a review, see ref. 3). Each of these techniques suffers from important disadvantages that may prevent their use for routine clinical pathology analysis of paraffinembedded specimens.

An all-optical instrument based on laser-induced breakdown spectroscopy (LIBS) was developed to create multi-elemental images of biological tissues. This technology was primarily developed with the aim of visualizing the distribution and kinetics of metal nanoparticles in the kidneys of laboratory animals. ${ }^{4,5}$

For this study, we selected relevant specimens with abnormal histopathological findings for which the pathologist could be interested in investigating the chemical composition of the unknown exogenous materials. These human formalin-fixed and paraffin-embedded specimens were histopathologically evaluated by conventional light microscopy examinations combined with adjacent LIBS multielemental imaging. Unlike standard pathology procedures, LIBS imaging successfully elucidated the chemical nature and distribution of exogenous agents, such as aluminum (Al) in a granuloma and a pseudolymphoma, and titanium (Ti), copper $(\mathrm{Cu})$, chromium (Cr) and tungsten (W) in lymph nodes and/or the skin.

This study demonstrated that LIBS imaging is a robust and versatile tool for identifying various foreign substances in paraffin-embedded specimens and therefore may aid pathologists in their daily diagnoses.

\section{Materials and methods}

\section{Patients}

For this study, the pathologists of our institutions selected retrospective formalin-fixed paraffinembedded specimens of interest, which were retrieved from the pathology departments of Grenoble University Hospital and Clermont-Ferrand University Hospital. Each sample was anonymized, and all unnecessary patient medical and personal data were deleted from the clinical reports. All experimental procedures were approved by the local Ethics Committees (CPP Sud-Est V).

\section{Histological Analysis}

Surface cut sections (5 $\mu$ m thickness) were prepared for optical microscopy, leaving a freshly cut surface of the block for complementary examination by LIBS. The pathology examination was performed after hematoxylin eosin saffron staining by expert pathologists. Hematoxylin eosin saffron sections were scanned using a field microscope with a motorized platform $(\times 2.5, \mathrm{NA}=0.085)$. The full view was acquired using MetaVue software.

\section{LIBS Imaging}

Elemental imaging by LIBS was performed directly on the paraffin block using a Nd:YAG laser operating at $1064 \mathrm{~nm}$ and pulsed at $10 \mathrm{~Hz}$ with a pulse energy of $4 \mathrm{~mJ} / \mathrm{shot}$. The complete technical settings were recently described in detail elsewhere. ${ }^{6}$ Briefly, a $\times 15$ magnification lens (LMM-15X-P01, Thorlabs, Germany) was used to focus the laser pulse, and the plasma emission was collected by two lens-fiber systems coupled to a Czerny-Turner spectrometer (Andor Technology). The spectrometer was equipped with an ICCD camera (Istar, Andor Technology) synchronized to the Q-switch of the laser. The paraffin-embedded specimens were placed onto the XYZ stage and scanned, pixel by pixel, with a resolution of $66 \mu \mathrm{m}$. The analyses were conducted at room temperature and under ambient pressure conditions. The laser pulse energy and its focalization onto the sample were strictly controlled. ${ }^{5}$ A specific spectral data treatment ${ }^{5}$ was applied to extract the signal intensity of the elements of interest and to convert it into a matrix displayed as an image by a false color scale. The typical spectral range used was from 247 to $330 \mathrm{~nm}$.

\section{Results}

\section{Local Cutaneous Reactions}

We performed LIBS multi-elemental analysis on cutaneous biopsies of persistent subcutaneous nodular lesions following vaccination or hyposensitization therapy. Cutaneous adverse reactions at the site of injection of vaccines or hyposensitization therapy containing $\mathrm{Al}$ salts are not uncommon. ${ }^{7}$ However, a variety of overlapping histopathological patterns mimic the patterns of other conditions such as granuloma, panniculitis, or pseudolymphoma. ${ }^{8,9}$

Phosphorus (P) or sodium (Na) is constitutive element of every cell in tissue; thus, creating elemental images of these so-called 'background elements' using LIBS allowed proper visualization of the global architecture of the tissue (Figure 1, Na). This result was confirmed by the good agreement between the elemental image of Na (Figure 1) and the histological images of the corresponding serial section stained with hematoxylin eosin saffron (Figure 1, left panels). Al salts are standard adjuvants in vaccines that might be the causative agents for allergy-related adverse events, which frequently are reported as temporary or persistent swellings at the site of injection. These pruritic nodules are the cause of considerable discomfort for patients. ${ }^{10}$ LIBS elemental analysis allowed direct in situ visualization of the levels of $\mathrm{Al}$ in a skin nodule at the site of a 
pediatric vaccination displaying the histopathological patterns of a granuloma (Figure 1, upper panels) and in a chronic inflammatory lesion at the site of hyposensitization therapy that revealed histopathological patterns that included nodular lymphohistiocytic infiltrate in the deep dermis (Figure 1, lower panels). In both cases, the localization of Al perfectly matched the areas containing histiocytes with intracytoplasmic bluish granules after hematoxylin eosin saffron staining. Such histiocytes with a granular cytoplasm might contain $\mathrm{Al}$ particles. ${ }^{2}$

\section{Pigmented Lymph Nodes}

The presence of node pigmentation on macroscopic evaluation is highly suggestive of metastatic malignant melanoma. However, the migration of exogenous ink particles from cutaneous tattoos is the most frequent cause for pigmented lymph nodes. ${ }^{11}$ Therefore, dark pigmented lymph nodes might represent a pitfall for surgeons or pathologists during lymph node biopsies or dissection procedures in the context of a suspicion of melanoma. a

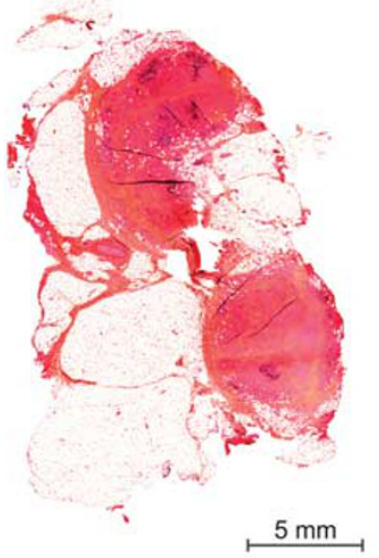

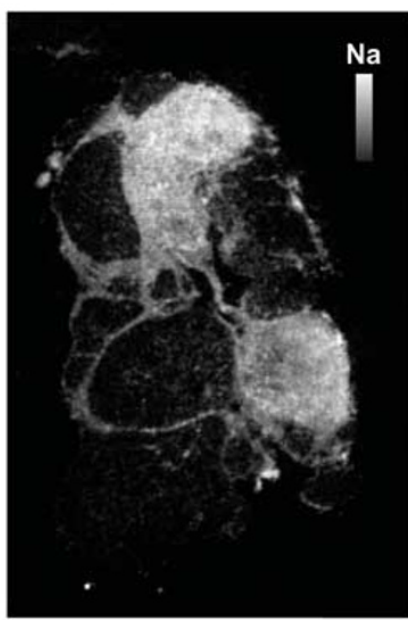
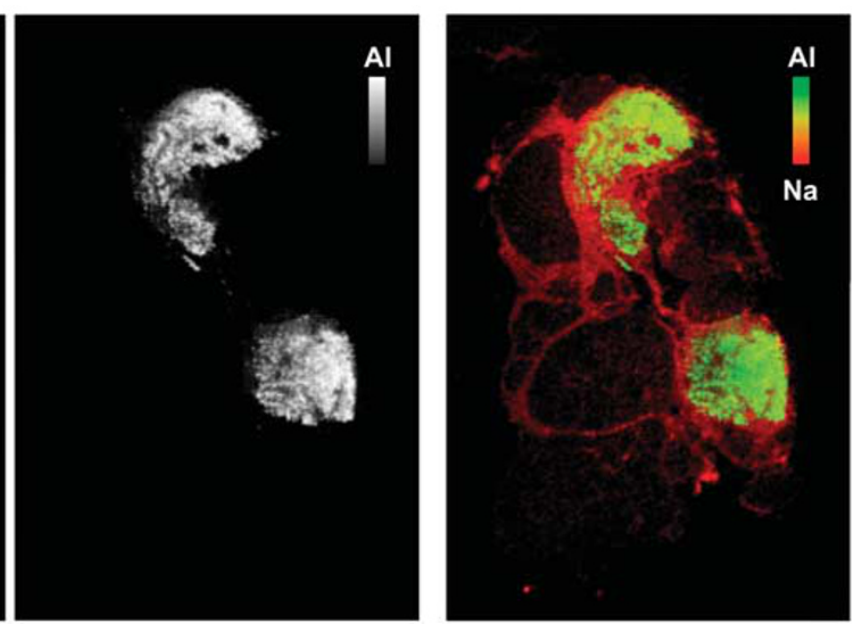

b
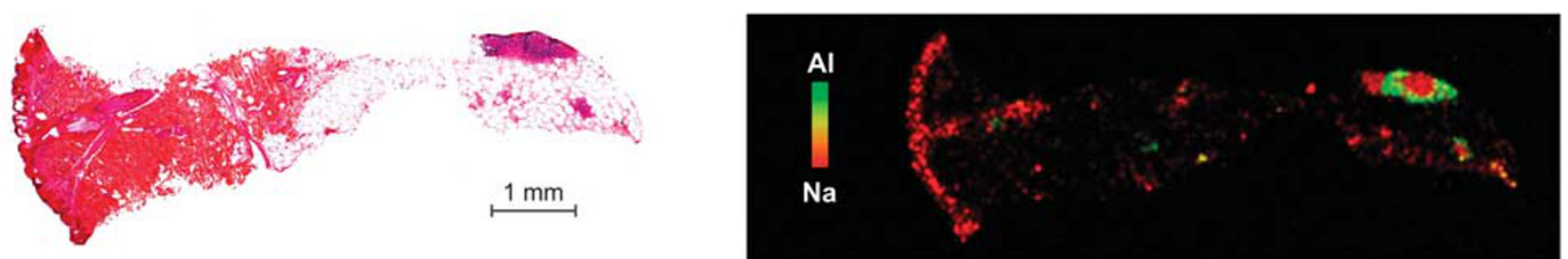

Figure 1 Aluminum (Al) accumulation in local skin reactions to Al-adsorbed immunotherapies. Histopathological morphology of a cutaneous granuloma (a) and a skin pseudolymphoma (b) with corresponding elemental images obtained after LIBS (right panels). LIBS analysis revealed high levels of $\mathrm{Al}$ (in green) in the immunohistiocytic areas. Sodium ( $\mathrm{Na}$, in red), enabled the visualization of the global tissue architecture. Scale bar: $1 \mathrm{~mm}$.
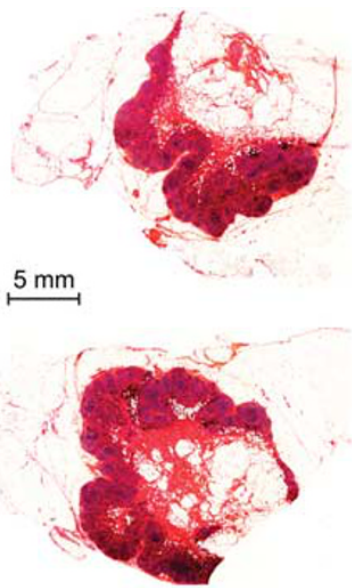
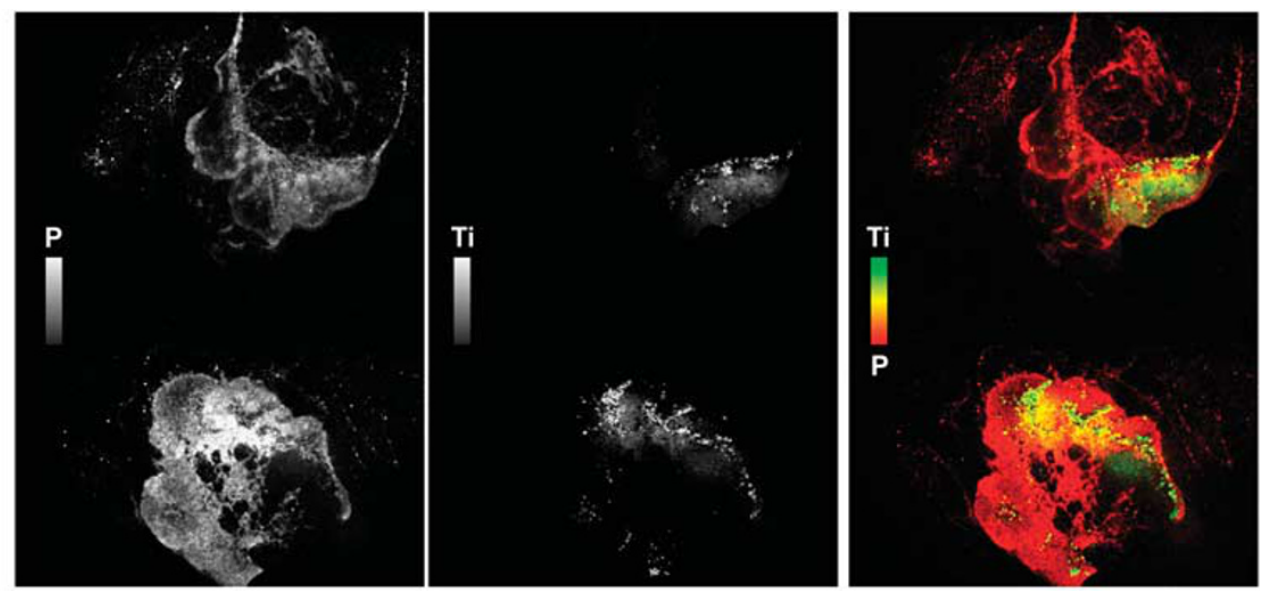

Figure 2 Titanium accumulation in a lymph node. Histopathological morphology of a pigmented lymph node (left panel) and the corresponding multi-elemental image obtained using LIBS (right panel). Numerous titanium particles (Ti, green) are present in the sinuses of the lymph node. Phosphorus (P, in red) was selected to represent the global tissue architecture of the lymph node. Scale bar: 1 mm. 
To illustrate such a challenge for pathologists, we analyzed a specimen from the external left leg of a male patient with a history of melanoma. The physicians performed a formal left inguinal lymph node dissection. During surgery, eight nodes were excised, including one node that was firm, black in color and macroscopically suspicious. The subsequent histopathological examination of the suspicious lymph node revealed the presence of black granular pigments as extracellular deposits between collagen bundles located in the sinuses of the lymph node, but not in the attached fatty tissue, along with rare histiocytes displaying small amounts of pigmented particles within their cytoplasm (Figure 2, left panel). Phosphorus analysis with LIBS allowed the proper visualization of the lymph node architecture (Figures 2). A LIBS multi-elemental investigation revealed the presence of elevated levels of $\mathrm{Ti}$ (Figure 2, Ti) in different areas of the lymph nodes that corresponded with the topography of the black pigment reported after histopathological examination. The pathologist's procedures demonstrated the absence of melanoma cells by the absence of any specific immuno-labeling against melanoma cells.

\section{Exogenous Particles After Surgery}

The presence of foreign material in tissues after surgery was also investigated. This aspect has been largely documented, ${ }^{12-17}$ and several exogenous substances, such as particles or debris, may originate from the release of components of surgical instruments, sutures, staples, implants, among other sources.

The analysis of a scar from a previous mastectomy (Figure 3) was chosen because the histopathological report revealed the presence of different resorptive processes in the fibrotic dermis, such as (i) multinucleated giant cells surrounding an anhistic foreign substance, (ii) histiocytic infiltrates with blue pigment in their cytoplasm, and (iii) a small aggregate of histiocytes or granulocytes with small periodic acidSchiff-positive granulations. LIBS images revealed the presence of a high Ti level in addition to the presence of $\mathrm{Cu}$ or localized $\mathrm{W}$ or $\mathrm{Cr}$ particles (Figure 3).

\section{Discussion}

Routine examination of tissue sections by light and polarizing microscopy, although adequate for most lesions, might fail to reveal sufficient information to allow specific diagnoses for many other lesions. In situ imaging of metals and foreign materials in cells and tissues is sometimes needed to document the nature of abnormal histopathological findings such as granules, vacuoles, pigments, or foreign bodies. The most classical approach is to perform histochemical staining of the tissue with chelators of the metal of interest. However, such chromogenic detection suffers from limitations such as poor sensitivity and/or specificity, limitations to certain metals, and restriction to only one element detected per dye. ${ }^{18}$

Alternative strategies have developed rapidly and are based on transmission electron microscopy coupled with energy dispersive X-ray analysis, ${ }^{19}$ laser ablation inductively coupled plasma mass spectrometry, ${ }^{20}$ secondary ion mass spectrometry, ${ }^{21}$ or synchrotron X-ray fluorescence microscopy. ${ }^{22}$ Although these techniques offer high performance in terms of sensitivity and/or spatial resolution, the high complexity of these instruments require qualified staff. In addition, the analytical time to scan full biopsies, such as that needed for collecting and analyzing the data, is generally substantial, and specific sample preparation procedures may hinder the analysis of large paraffin-embedded specimens. ${ }^{18}$ Consequently, these disadvantages prevent the use of such equipment for routine medical diagnosis, and pathologists are currently lacking available technologies with convenient procedures to investigate the chemical composition of exogenous materials.

Using insights gained in analytical sciences and preclinical research, ${ }^{4,5,23}$ we demonstrated that LIBS imaging enabled the elemental analyses of largesized samples embedded in paraffin in a relatively simple, inexpensive, and reliable manner.

Although slightly destructive to the sample, LIBS analysis has the ability to identify almost every element from the periodic table, including those with atomic numbers lower than $11{ }^{24}$ By contrast, light elements with low atomic numbers are difficult to detect with energy dispersive X-ray analysis or laser ablation inductively coupled plasma mass spectrometry techniques. LIBS instruments perform fast, full scans of large-sized paraffin-embedded specimens with a typical time of analysis of $3 \mathrm{~h}$ for scanning an $\sim 6 \mathrm{~cm}^{2}$ surface with $10 \mathrm{~Hz}$ frequency lasers or only $20 \mathrm{~min}$ with $100 \mathrm{~Hz}$ lasers. This alloptical benchtop instrument works at ambient pressure and temperature. As direct comparison between multi-elemental images and microscopy images are necessary for pathologists, we took advantage of serial sectioning procedures in studying hematoxylin eosin saffron sections for histopathology with the corresponding adjacent paraffin block for LIBS analysis.

LIBS detected the presence of different metals, such as $\mathrm{Al}$ in a cutaneous granuloma and a skin pseudolymphoma, as well as the presence of Ti, W, $\mathrm{Cr}$, and $\mathrm{Cu}$ in lymph nodes and/or in the skin, directly in the paraffin-embedded specimens of origin.

For both the granuloma and the pseudolymphoma, the histopathological analysis combined with the pathologists' experience and the clinical history strongly suggested an immune reaction against one of the components of the vaccines, possibly, if not chiefly, Al salts. Cutaneous adverse reactions to $\mathrm{Al}$ salts are well described in the literature.$^{7,8}$ Here the 

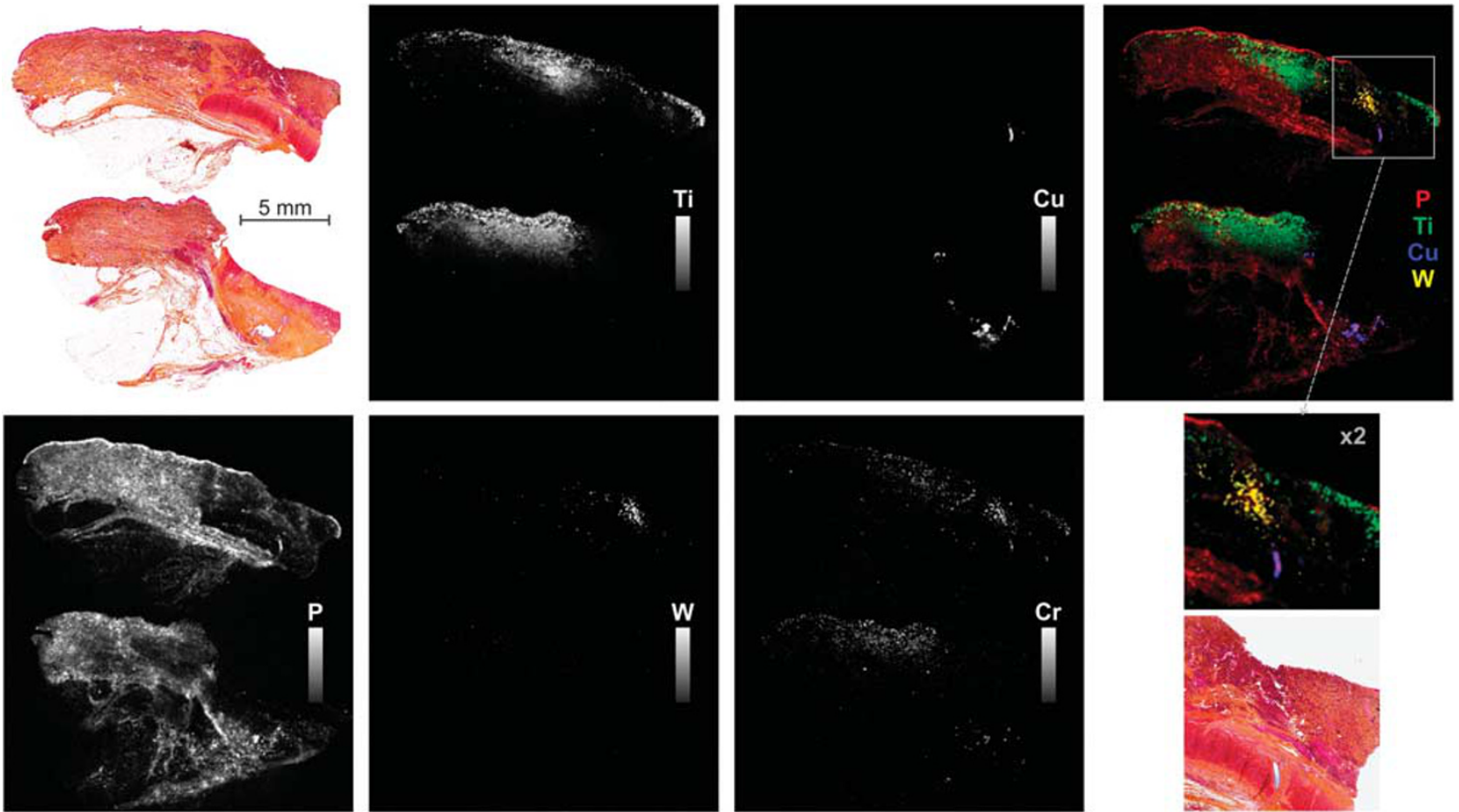

Figure 3 Metal particles in human skin. Histopathological morphology of a cutaneous scar from a mastectomy (hematoxylin eosin saffron, $\times 10$; left upper panel) and at higher magnification (right lower panel) along with the corresponding multi-elemental images obtained with LIBS. Phosphorus (P) was selected to represent the tissue (red), and titanium (Ti) is shown in green. In addition, copper (Cu) is shown in blue and tungsten (W) is shown in yellow. The presence of chromium (Cr) particles is also shown. Scale bar: $1 \mathrm{~mm}$.

presence of $\mathrm{Al}$ was inferred from both the clinical context (ie, history of vaccination/hyposensitization or delayed cutaneous reactions at the site of injection) and histopathological criteria (ie, the presence of deep dermal or subcutaneous nodules or histiocytes containing bluish granules in their cytoplasm). In contrast to these presumptive approaches, the genuine documentation of the presence of high levels of $\mathrm{Al}$ in the granulomatous areas was enabled by complementary multi-elemental LIBS imaging.

Alternatively, the direct identification of $\mathrm{Al}$ in tissues was previously performed by different methods, such as histochemistry with solochrome azurine stains (not specific, interference from iron ${ }^{25}$ ), pentahydroxyflavone staining for $\mathrm{Al}$ deposits (necessitating hydrochloric acid pre-treatment and fluorescence microscopy), or complex electron probe microanalysis conducted on an electron microscope equipped with a radiographic spectrometer. ${ }^{8,9,26}$ These alternative strategies for determining the presence of $\mathrm{Al}$ are tedious. Consequently, they are rarely, if ever, performed routinely in clinical pathology laboratories.

In addition to $\mathrm{Al}$ immune reactions, we anticipate the important benefit of LIBS imaging to be the screening of other exogenous elements involved in cutaneous reactions, such as silica, ${ }^{27,28}$ or for any granulomatous reaction, especially foreign-body granulomas and sarcoidal granulomas. ${ }^{1}$ Interestingly, routine light microscopy and polarizing microscopy do not identify foreign material in sarcoidal granulomas, although contact allergies to beryllium, ${ }^{29}$ nickel, Ti, zirconium, or other metals have been reported. ${ }^{2}$ LIBS multi-elemental imaging could also be of interest for better understanding the etiology of histopathological patterns that are frequently caused by the presence of exogenous substances that induce granulomatous reactions, as well as for better understanding the respiratory diseases related to environmental and occupational exposure to mineral particles, metals, and dust. ${ }^{30}$

The presence of black pigments similar to melanin pigments could mimic melanoma metastasis. ${ }^{31}$ LIBS analysis revealed the chemical nature of the dark pigments in node samples, which consisted of $\mathrm{Ti}$ particles. Several reports have described the coincidental finding of pigmented lymph nodes due to the migration of tattoo pigments simulating regional dissemination of metastatic melanoma cells. ${ }^{11,32}$

In such cases of lymph node discoloration, the rare, but significant, incidents of coincidental findings between benign pigment collections in nodes and the dissemination of melanoma should necessitate meticulous immunohistopathological analysis of the suspected metastatic tissues. Standard histopathological procedures are generally sufficient to demonstrate the presence of melanoma macro- or micro-metastasis. ${ }^{33}$ However, complementary elemental investigation could be helpful in litigation cases or to gain a complete understanding of the underlying etiology of a lesion. 
Multi-elemental imaging of scar tissue revealed the presence of several Ti particles as well as localized W and Cr particles. Granulomas might develop adjacent to surgical areas or scars, and the presence of metal particles originating from components of the instruments used during the surgery or from the implants or prostheses themselves is well documented. ${ }^{12-17,34,35}$ Therefore, particles or debris of all sizes, which are generally pigmented and birefringent or not under polarizing light microscopes, are regularly observed by on-duty pathologists. ${ }^{35}$ Some assumptions can be made about the presence of these foreign metals. An analysis of diathermy instruments showed that cutting loops from microsurgical needle electrodes are made of pure $\mathrm{W},{ }^{36}$ whereas Ti may originate from metal $\mathrm{Ti}$ or Ti-containing alloys used in biocompatible prosthetic devices, ${ }^{37}$ pacemaker implants, ${ }^{38}$ ear piercings, ${ }^{1}$ or from drainage of the ink particles from a nearby tattoo. ${ }^{39}$

Notably, tattoo pigments are frequently found in the skin and/or lymph nodes, in which they might induce sarcoidal or foreign-body granulomas or pseudolymphomas. ${ }^{2}$ According to the color and the composition of the inks that were used, one or several pigmented granules might be found in the tissue. Elemental analysis of specimens with tattoo pigments may reveal the presence of Ti (white inks and lightening agents), mercury, iron, cadmium or selenium (red inks), cadmium-sulfur (yellow inks), chromium (green inks), cobalt and Ti (blue inks), or manganese (purple inks). ${ }^{1}$ Therefore, recording the presence of decorative tattoos is crucial. ${ }^{31}$

The presence of overlapping histopathological patterns can lead to misdiagnosis or insufficient diagnosis. In a study of 15 cases of suspected amalgam tattoos, multi-elemental analysis of pigmented materials with an energy dispersive X-ray microanalyzer helped to differentiate the fine spherical golden brown granules of amalgam from the remarkably similar hemosiderin and melanin granules. ${ }^{40}$ Immunohistochemistry performed with routine or special stains is not always reliable for differentiating exogenous from endogenous substances, and elemental investigations should be considered by the pathologists to fulfill their obligation of returning a diagnosis that is as complete and accurate as possible to the clinician and the patient. $^{41}$

Because the appropriate clinical management of patients depends on a rapid and accurate diagnosis and the morphological features among the items of a differential diagnosis may be confounding, the definitive diagnosis could be confirmed by complementary LIBS in situ elemental imaging for the determination of the specific elemental composition of the foreign material in the specimen.

In addition to applications for exogenous agents, a need exists for in situ elemental imaging of a steadily growing number of identified diseases characterized by metal imbalance in cells and tissues (eg, metal overload diseases, neurodegenerative diseases, or even cancers). ${ }^{3}$ LIBS could provide robust, valuable complementary elemental information to unravel the pathogenesis of such metal-related diseases.

In summary, the combination of standard immunohistochemistry procedures with LIBS multielemental imaging is a promising approach for routine investigations of the chemical composition of foreign substances contained in paraffinembedded specimens.

\section{Acknowledgments}

We are thankful for research funding from the ITMO Cancer et ITMO Technologies pour la santé de l'alliance nationale pour les sciences de la vie et de la santé (AVIESAN), the Institut National du Cancer (INCa), and INSERM within the project LAST (\#PC201513).

\section{Disclosure/conflict of interest}

The authors declare no conflict of interest.

\section{References}

1 Molina-Ruiz AM, Requena L. Foreign body granulomas. Dermatol Clin 2015;33:497-523.

2 Requena L, Cerroni L, Kutzner H. Histopathologic patterns associated with external agents. Dermatol Clin 2012;30:731-748 vii.

3 McRae R, Bagchi P, Sumalekshmy S, et al. In situ imaging of metals in cells and tissues. Chem Rev 2009;109:4780-4827.

4 Gimenez Y, Busser B, Trichard F, et al. 3D imaging of nanoparticle distribution in biological tissue by laserinduced breakdown spectroscopy. Sci Rep 2016;6: 29936.

5 Sancey L, Motto-Ros V, Busser B, et al. Laser spectrometry for multi-elemental imaging of biological tissues. Sci Rep 2014;4:6065.

6 Moncayo S, Trichard F, Busser B, et al. Multi-elemental imaging of paraffin-embedded human samples by laserinduced breakdown spectroscopy. Spectrochim Acta B At Spectrosc 2017;133:40-44.

7 Bergfors E, Bjorkelund C, Trollfors B. Nineteen cases of persistent pruritic nodules and contact allergy to aluminium after injection of commonly used aluminiumadsorbed vaccines. Eur J Pediatr 2005;164:691-697.

8 Chong H, Brady K, Metze D, et al. Persistent nodules at injection sites (aluminium granuloma)—Clinicopathological study of 14 cases with a diverse range of histological reaction patterns. Histopathology 2006;48: 182-188.

9 Maubec E, Pinquier L, Viguier M, et al. Vaccinationinduced cutaneous pseudolymphoma. J Am Acad Dermatol 2005;52:623-629.

10 Bergfors E, Lundmark K, Nystrom Kronander U. A child with a long-standing, intensely itching subcutaneous nodule on a thigh: an uncommon (?) reaction to commonly used vaccines. BMJ Case Rep 2013; doi:10.1136/bcr-2012-007779. 
11 Friedman $\mathrm{T}$, Westreich $\mathrm{M}$, Mozes SN, et al. Tattoo pigment in lymph nodes mimicking metastatic malignant melanoma. Plast Reconstr Surg 2003;111:2120-2122.

12 Flatebo RS, Hol PJ, Leknes KN, et al. Mapping of titanium particles in peri-implant oral mucosa by laser ablation inductively coupled plasma mass spectrometry and high-resolution optical darkfield microscopy. J Oral Pathol Med 2011;40:412-420.

13 Lobinski R, Moulin C, Ortega R. Imaging and speciation of trace elements in biological environment. Biochimie 2006;88:1591-1604.

14 Torgersen S, Gjerdet NR, Erichsen ES, et al. Metal particles and tissue changes adjacent to miniplates. A retrieval study. Acta Odontol Scand 1995;53:65-71.

15 Koppang HS, Roushan A, Srafilzadeh A, et al. Foreign body gingival lesions: distribution, morphology, identification by X-ray energy dispersive analysis and possible origin of foreign material. J Oral Pathol Med 2007;36:161-172.

16 Nihon-Yanagi Y, Ishiwatari T, Otsuka Y, et al. A case of postoperative hepatic granuloma presumptively caused by surgical staples/clipping materials. Diagn Pathol 2015;10:90.

17 Lohmann CH, Meyer H, Nuechtern JV, et al. Periprosthetic tissue metal content but not serum metal content predicts the type of tissue response in failed smalldiameter metal-on-metal total hip arthroplasties. J Bone Joint Surg Am 2013;95:1561-1568.

18 Susnea I, Weiskirchen R. Trace metal imaging in diagnostic of hepatic metal disease. Mass Spectrom Rev 2016;35:666-686.

19 Scimeca M, Orlandi A, Terrenato I, et al. Assessment of metal contaminants in non-small cell lung cancer by EDX microanalysis. Eur J Histochem 2014;58:2403.

20 Urgast DS, Beattie JH, Feldmann J. Imaging of trace elements in tissues: with a focus on laser ablation inductively coupled plasma mass spectrometry. Curr Opin Clin Nutr Metab Care 2014;17:431-439.

21 Bich C, Touboul D, Brunelle A. Biomedical studies by TOF-SIMS imaging. Biointerphases 2014;10:018901.

22 Chao W, Harteneck BD, Liddle JA, et al. Soft X-ray microscopy at a spatial resolution better than $15 \mathrm{~nm}$. Nature 2005;435:1210-1213.

23 Sancey L, Kotb S, Truillet C, et al. Long-term in vivo clearance of gadolinium-based AGuIX nanoparticles and their biocompatibility after systemic injection. ACS Nano 2015;9:2477-2488.

24 Qiao S, Ding Y, Tian D, et al. A review of laser-induced breakdown spectroscopy for analysis of geological materials. Appl Spectrosc Rev 2015;50:1-26.

25 Fernandez-Martin JL, Menendez P, Acuna G, et al. Staining of bone aluminium: comparison between aluminon and solochrome azurine and their correlation with bone aluminium content. Nephrol Dial Transplant 1996;11(Suppl 3):80-85.
26 Culora GA, Ramsay AD, Theaker JM. Aluminium and injection site reactions. J Clin Pathol 1996;49: 844-847.

27 Shelley WB, Hurley HJ. The pathogenesis of silica granulomas in man: a non-allergic collidial phenomenon. J Invest Dermatol 1960;34:107-123.

28 Mowry RG, Sams WM Jr., Caulfield JB. Cutaneous silica granuloma. A rare entity or rarely diagnosed? Report of two cases with review of the literature. Arch Dermatol 1991;127:692-694.

29 Berlin JM, Taylor JS, Sigel JE, et al. Beryllium dermatitis. J Am Acad Dermatol 2003;49:939-941.

30 Goldyn SR, Condos R, Rom WN. The burden of exposure-related diffuse lung disease. Semin Respir Crit Care Med 2008;29:591-602.

31 Jack CM, Adwani A, Krishnan H. Tattoo pigment in an axillary lymph node simulating metastatic malignant melanoma. Int Semin Surg Oncol 2005;2:28.

32 Chikkamuniyappa S, Sjuve-Scott R, Lancaster-Weiss K, et al. Tattoo pigment in sentinel lymph nodes: a mimicker of metastatic malignant melanoma. Dermatol Online J 2005;11:14.

33 Peterson SL, Lee LA, Ozer K, et al. Tattoo pigment interpreted as lymph node metastasis in a case of subungual melanoma. Hand (N Y) 2008;3:282-285.

34 Albores-Saavedra J, Vuitch F, Delgado R, et al. Sinus histiocytosis of pelvic lymph nodes after hip replacement. A histiocytic proliferation induced by cobaltchromium and titanium. Am J Surg Pathol 1994;18: 83-90.

35 Peoc'h M, Moulin C, Pasquier B. Systemic granulomatous reaction to a foreign body after hip replacement. $\mathrm{N}$ Engl J Med 1996;335:133-134.

36 Henry L, Wagner B, Faulkner MK, et al. Metal deposition in post-surgical granulomas of the urinary tract. Histopathology 1993;22:457-465.

37 Fage SW, Muris J, Jakobsen SS, et al. Titanium: a review on exposure, release, penetration, allergy, epidemiology, and clinical reactivity. Contact Dermatitis 2016;74:323-345.

38 Viraben R, Boulinguez S, Alba C. Granulomatous dermatitis after implantation of a titanium-containing pacemaker. Contact Dermatitis 1995;33:437.

39 Willardson HB, Kobayashi TT, Arnold JG, et al. Diffuse urticarial reaction associated with titanium dioxide following laser tattoo removal treatments. Photomed Laser Surg 2017;35:176-180.

40 Hartman LC, Natiella JR, Meenaghan MA. The use of elemental microanalysis in verification of the composition of presumptive amalgam tattoo. J Oral Maxillofac Surg 1986;44:628-633.

41 Daley TD, Gibson D. Practical applications of energy dispersive X-ray microanalysis in diagnostic oral pathology. Oral Surg Oral Med Oral Pathol 1990;69: 339-344. 